\title{
Behavioral modeling of Digitally Adjustable Current Amplifier
}

\author{
J. Polak, L. Langhammer, and J. Jerabek
}

\begin{abstract}
This article presents the digitally adjustable current amplifier (DACA) and its analog behavioral model (ABM), which is suitable for both ideal and advanced analyses of the function block using DACA as active element. There are four levels of this model, each being suitable for simulation of a certain degree of electronic circuits design (e.g. filters, oscillators, generators). Each model is presented through a schematic wiring in the simulation program OrCAD, including a description of equations representing specific functions in the given level of the simulation model. The design of individual levels is always verified using PSpice simulations. The ABM model has been developed based on practically measured values of a number of DACA amplifier samples. The simulation results for proposed levels of the $\mathrm{ABM}$ model are shown and compared with the results of the real measurements of the active element DACA.
\end{abstract}

Keywords-Digitally adjustable current amplifier, controllable gain, DACA, ABM model, PSpice, modeling.

\section{INTRODUCTION}

Many scientific articles in professional journals describe the design of frequency filters with a clear objective to create a universal filter e.g. with the lowest possible power consumption requirements. Those articles often focus on the design of filters working in the current mode $(\mathrm{CM})$, with current considered as the input and output variable. The second option for the filter design is using the voltage-mode (VM) approach, with voltage considered as the input and output variable. These two modes can also be combined. When designing frequency filters, we have available active elements and their various modifications working on different principles, such as operational amplifiers (OPA), operational transconductance amplifiers (OTA), voltage conveyors (VC), current conveyors (CC), current feedback amplifiers (CFA), voltage followers (VF), current followers (CF), etc. [1]

An important parameter in the selection of an active element is the possibility of electronic control of the specific

Manuscript received January 13, 2015, revised February 16, 2015.

J. Polak Faculty of Electrical Engineering and Communication,

Brno University of Technology, Technicka 12, 61600 Brno, Czech Republic (e-mail: xpolak24@stud.feec.vutbr.cz)

L. Langhammer Faculty of Electrical Engineering and Communication, Brno University of Technology, Technicka 12, 61600 Brno, Czech Republic (e-mail: xlangh01@ @stud.feec.vutbr.cz)

J. Jerabek Faculty of Electrical Engineering and Telecommunication, Brno University of Technology, Technicka 12, 61600 Brno, Czech Republic (e-mail: jerabekj@feec.vutbr.cz) parameter (gain, input impedance, etc.) by means of DC current or DC voltage. Controllable active element consists of simpler uncontrollable and controllable active elements. One of the fundamental active elements is the current differencing buffered amplifier (CDBA) [2], which can be created from two active elements current feedback amplifier (CFA) [2], [3], implemented for example through AD844 [4], [5].

By replacing the voltage buffer in the active element CDBA by an balanced-output operational transconductance amplifier (BOTA), an active element CDTA [6], [7] is created. The function of this circuit shows that it is possible to regulate the gain using transconductance and that it is suitable for use in construction of filters working in the current mode [6]. The current follower transconductance amplifier (CFTA) [8], with only one current input, is based on a similar principle as CDTA. CFTA transconductance is controllable and it is suitable for the construction of filters in the current mode.

Other parameters of active elements are often also electronically controlled, usually using DC current. The active element current-controlled current conveyor transconductance amplifier (CCCCTA) [9] is based on the principle of current conveyor transconductance amplifier (CCTA) presented in [10], but there is a controllable input impedance at input $\mathrm{x}$, which can be controlled by current, as shown in [9]. CCCCTA may be created using a current controlled current conveyor (CCCII) [11] and OTA. By combination of these active elements, we can also create a current controlled current follower transconductance amplifier (CCCFTA) [12]. In that case the input y of CCCII is grounded and the OTA replaced by a BOTA. This active element is again electronically controllable through current as for CCCCTA.

Another group of controllable active elements are the digitally controlled active elements. Their properties are controlled by digital $n$-bit word. One of the digitally controlled active elements is the digitally controlled current conveyor (DCCC) [13]. This element is digitally controlled using current division network (CDN) [13], wherein the number of control bits is determined by the number of current division cells (CDC) [13]. Another possibility of creating $\mathrm{CDC}$ is based on the use of the unity-gain current amplifier with very low-resistance input terminal [14] and a current divider circuit. This principle is the basis for the digital control of the element digitally controlled current follower (DC-CF) [15].

The active element controlled using the digital word, presented in this article, is the digitally adjustable current amplifier (DACA) [16], [17]. It has been recently developed by the Department of Telecommunications of the Brno 
University of Technology in cooperation with the company ON Semiconductor, based on verified technology in CMOS $0.35 \mu \mathrm{m}$, suitable for analogue signal processing. The active element DACA can be used for instance in circuits of frequency filters, oscillators and function generators [18][21]. In this paper we present newly designed ABM models [22] of the element DACA in four levels (zero, first, second, third level) in PSpice. As it is discussed in section III, the zero model describes only basic (ideal) behavior of the DACA. The first model is designed so that it reflects the inputs and outputs resistances and real values of gain, the second model is similar to the first level and it is supplemented by other functions to show the frequency dependence of input and output impedance values for different gains. The third level takes into account the real current limits of the inputs and outputs. This is particularly suitable when simulating complex electronic circuits containing different types of elements, sub-circuits and devices, require different levels of modelling for different circuits and therefore make it impractical to include all the functions into a single level of simulation model [23]. Dividing the model into multiple levels, a more detailed examination of the desired characteristics of the analysed circuit can be provided.

\section{FUNCTIONAL PRINCIPLES OF DACA}

The active element DACA (Fig. 1a) consists of differential current inputs and outputs. Inputs are of low impedance and outputs high impedance characters. The current gain can be adjusted with unity step from 1 to 8 of the input current in the non-differential connection. In the differential connection, the gain can be adjusted with 2-unit step from 2 to 16 of the input current. The gain control is realized using a 3-bit bus (referred as CTR in Fig. 1a, b). The schematic symbol of the active element DACA is shown in Fig. 1a.

\section{DACA}

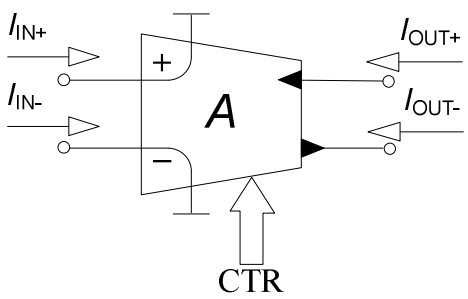

Fig. 1a. Schematic symbol of DACA element

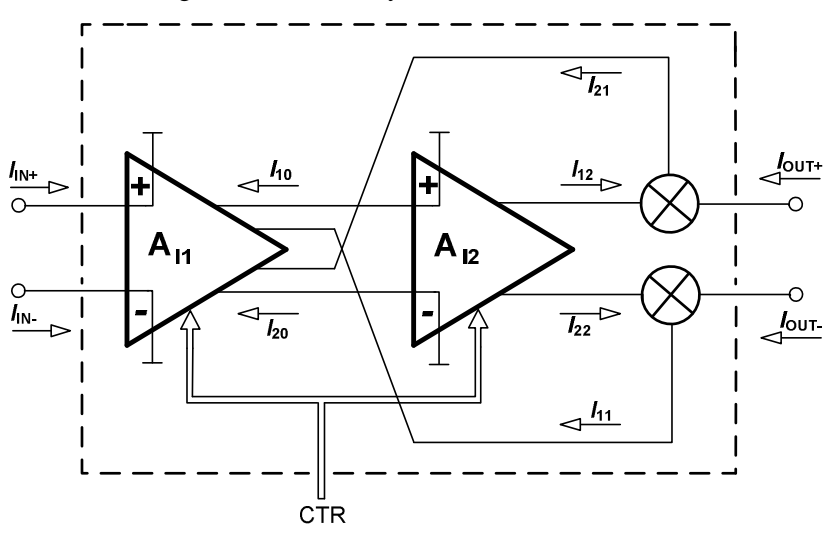

Fig. 1b. Block internal structure of DACA element

Figure $1 \mathrm{~b}$ shows the internal structure of the circuit. It is based on a pair of digitally controlled current amplifiers.
The relationship between internal and external currents is expressed by following equations:

$$
\begin{array}{clrl}
I_{10}=I_{I N+}, & \text { (1) } & I_{20}=I_{I N-}, \\
I_{11}=A_{I 1} I_{I N+}, & \text { (2) } & I_{21}=A_{I 1} I_{I N-}, \\
I_{12}=A_{I 2} I_{10}, & \text { (3) } & I_{22}=A_{I 2} I_{20} .
\end{array}
$$

The resulting function of the circuit can be summarized as follows:

$I_{D I F_{-} I N}=\left(I_{I N+}-I_{I N-}\right)$,

$I_{\text {DIF_oUT }}=\left(I_{\text {OUT }+}-I_{\text {OUT- }}\right)$,

$I_{\text {OUT+ }}=A_{I}\left(I_{I N+}-I_{I N-}\right)$,

$I_{\text {OUT- }}=-A_{I}\left(I_{I N+}-I_{I N-}\right)$,

$I_{\text {DIF_oUT }}=2 A_{I} I_{D I F_{-} I N}$,

with $A_{I}$ as the current gain of the active element DACA and $I_{\text {DIF_IN }}, \mathrm{I}_{\text {DIF_OUT }}$ as the input and output differential currents.

\section{ABM MODEL OF DACA}

The ABM model for the active element DACA was developed to create the best approximation of circuit characteristics considered in the simulations during the design of electrical circuits that use this active element. The ABM model consists of several levels, each level adds certain undesired, but realistic, characteristics (e.g. input impedance, output impedance, frequency dependence, DC limitations) corresponding to the real properties of the DACA element. The use of individual levels in the model design allows us to identify and analyse how the adverse effects influence the function of the circuit.

\section{A. Zero level macro model}

The zero level of the model represented in Fig. 2 is designed so that the element behaves as ideal. The use of the active element is not affected by any adverse effects. Parts labelled F1-F4 represent current-controlled current sources and their task is to simulate ideal transmissions of the controllable current amplifier DACA using specified functions. The following equations show the internal current source F3 function, equation (12), into which are substituted values from equation (14) following the variation of the input variable gain. The current source F4 is represented by equation (13), into which are substituted values from equation (15), again depending on the variation of gain. Equations (12-15) are control functions directly used for the PSpice simulation program.

GAIN=\{F3@funcref(@gain) $\}$,

GAIN = \{F4@funcref(@gain) $\},$

.FUNC F3@funcref $(\mathrm{g})=$

\{TABLE (g, 1,1,2,2,3,3,4,4,5,5,6,6,7,7,8,8)\},

.FUNC F4@funcref(g)

$=\{$ TABLE $(\mathrm{g}, 1,-1,2,-2,3,-3,4,-4,5,-5,6,-6,7$, 
DACA 0 level

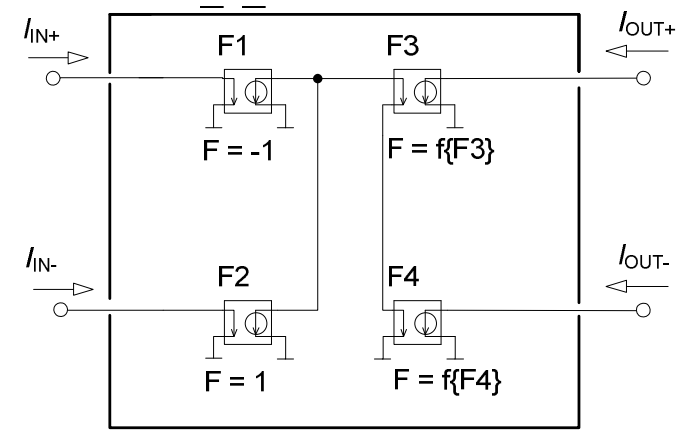

Fig. 2. Model of the zero level of DACA element

\section{B. First level macro model}

The first level of the DACA simulation model (Fig. 3) is designed so that it reflects the inputs and outputs resistances and real values of gain. Input resistance (resistors $\mathrm{R}_{1}$ and $\mathrm{R}_{2}$ ) is the same for all values of gain. The values of the output resistances (resistors $R_{3}$ and $R_{4}$ ) are chosen depending on the selected value gain. The gain of components F3 and F4 already corresponds to the real measured gain values of the element DACA. Into equations (12) and (13) are substituted values from equations (18) and (19). For resistors $R_{3}$ and $R_{4}$ the value of resistance (variable IMPl) changes with the variation of gain according to equations (16) and (17), which results in variation in the output impedance depending on the selected gain. The resistor $R_{3}$ is represented by equation (16), into which are substituted values from equation (20). Similarly, resistor $R_{4}$ is represented by equation (17), into which are substituted values from equation (21), in both cases depending on the value of specific gain.

$I M P 1=\{$ Fr3@funcref $(@ g a i n)\}$,

$I M P 1=\{F r 4 @ f u n c r e f(@ g a i n)\}$,

.FUNCF3@funcref $(g)$

$=\{T A B L E(g, 1,0.779,2,1.594,3,2.489,4,3.299,5$,

$4.133,6,4.936,7,5.814,8,6.609)\}$,

.FUNCF4@funcref $(g)$

$=\{T A B L E(g, 1,-0.801,2,-1.633,3,-2.55,4$,

$-3.378,5,-4.232,6,-5.054,7,-5.951,8,-6.763)\}$,

.FUNCFr3@funcref $(g)$

$=\{T A B L E(g, 1,72762.57,2,34139.24,3,21155.61$,

4,15713.62,5,11815.86,6,9862.1,7,8323.96,

8,7236.25)\},

.FUNCFr4@funcref $(g)$

$=\{$ TABLE $(g, 1,72748.87,2,34119.5,3,21390.12$,

$4,15890.39,5,12044.26,6,10046.73,7,8430.3$,

$8,7335.59)\}$.
DACA 1st level

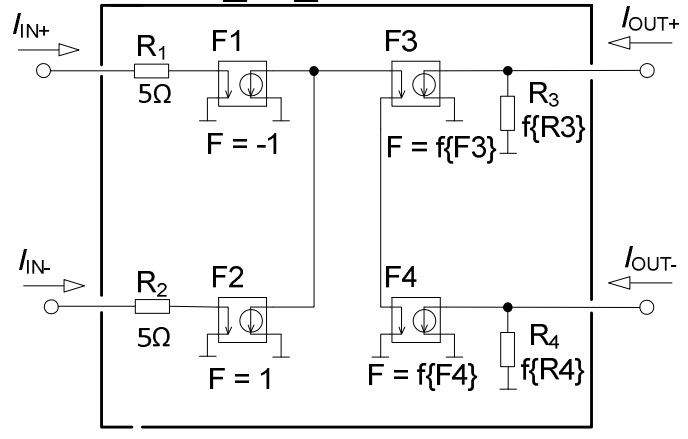

Fig. 3. Model of the first level of DACA element

Equations (18) and (19) show that at higher gain values the output current values differ more significantly from the ideal current values specified in equations (14) and (15). Increasing the gain value also reduces the value of the output impedance. This statement is supported by the output resistance values specified in equations (20) and (21) for both outputs depending on the gain. The value of the output impedance is the highest at gain equal to 1, approximately $72 \mathrm{k} \Omega$.

\section{Second level macro model}

The design of the second level of the simulation model presented in Fig. 4 is similar to the first level and is completed by other functions to show the frequency dependence of input and output impedance values for different gains. The frequency dependence is simulated using capacitors $\mathrm{C}_{3}$ and $\mathrm{C}_{4}$, whose functions are given in equations (22) and (23). These equations are then supplemented by values from equations (24) and (25) depending on selected gain level.

F_formula=\{FC3@funcref(@gain) $\}$,

F_formula = $F C 4 @$ funcref(@gain) $\}$,

.FUNCFC3@funcref $(g)$

$=\{T A B L E(g, 1,12.2 p, 2,12.6 p, 3,12.7 p, 4,12.9 p$,

$5,13.4 p, 6,13.6 p, 7,13.6 p, 8,13.4 p)\}$,

.FUNCFC4@funcref $(g)$

$=\{T A B L E(g, 1,12.5 p, 2,12.4 p, 3,13.1 p, 4,13.3 p$,

$5,13.7 p, 6,13.9 p, 7,13.7 p, 8,13.5 p)\}$.

DACA_2nd_level

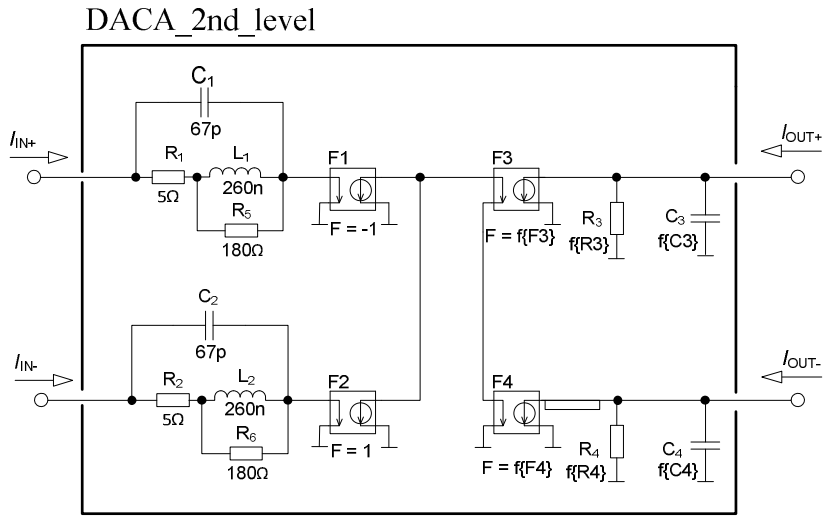

Fig. 4. Model of the second level of DACA element 
As an example, Fig. 5 shows the frequency dependence of the output current at output OUT+ for the different gain levels. The results shown in the graph were obtained by excitation of input $\mathrm{IN}+$ by input current $I_{I N+}=1 \mathrm{~mA}$ with connected load $50 \Omega$ on OUT+. Approximately until reaching the frequency of $10 \mathrm{MHz}$, the output current at output OUT+ (Fig. 5) and OUT- (similar results to OUT+) are constant. At higher frequencies, there is a decrease in the ability of the amplifying gain values, reflected in a decrease of both output currents (OUT+ and OUT-).

Similar frequency dependence can be seen in the graph in Fig. 6, showing the frequency dependence of the output impedance for output OUT+. The decrease in the output impedance starts at gain value equal to 1 already at a frequency of $15 \mathrm{kHz}$, whereas at gain value equal to 8 it only starts at a frequency of $500 \mathrm{kHz}$.

Fig. 7 shows the frequency dependence of the input impedance. The input impedance of the amplifier is constant $Z_{I N}=5 \Omega$ for all values of gain and its frequency dependence is reflected in slight growth from $1 \mathrm{MHz}$ and then a steep increase from $10 \mathrm{MHz}$.

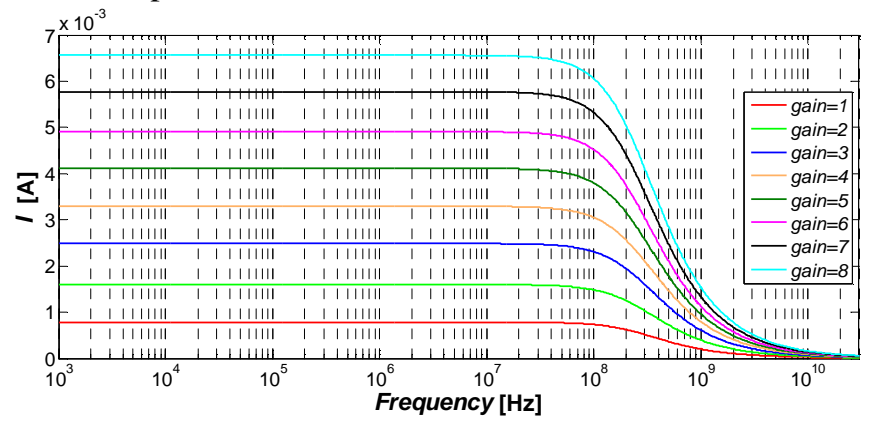

Fig. 5. Output current $\mathrm{I}_{\mathrm{OUT}}$ for each value of gain

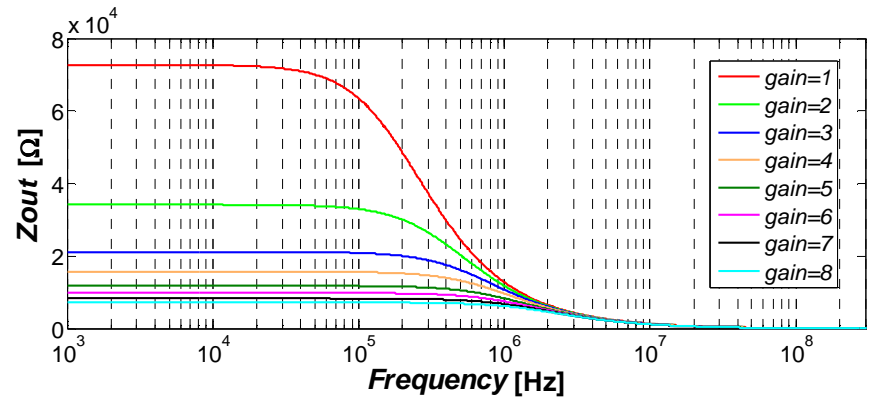

Fig. 6. Output impedance $\mathrm{Z}_{\mathrm{OUT}+}$ for each value of gain

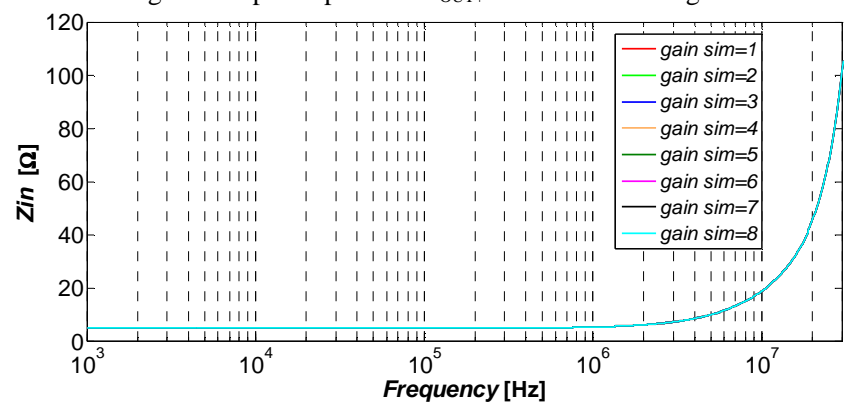

Fig. 7. Input impedance $\mathrm{Z}_{\mathrm{IN}+}$ for each value of gain

\section{Third level macro model}

The third level of the simulation model represented in Fig. 8 takes into account the real current limits of inputs and outputs. Input current limit is defined by inserting the function represented by equation (26) into the element F1 and the equation (27) into the element F2 of the proposed model. Upper and lower input current limit is fixed by using variables $H I(H i g h)=300 \mu \mathrm{A}$ and $L O($ Low $)=-300 \mu \mathrm{A}$.
The output current limit is realized by inserting function (26) into elements F5 and F6. Values of variables HI and LO from equations (28) and (29) are then substituted into equation (26). Subsequently, in equation (28) values are added depending on the currently selected gain (variable gain) from equation (31), which sets the current limit of element F5. To set the element F6 we use equation (26) and add to it the values of current HI and LO from equations (30) and (29). Then we assign values to load depending on the currently selected gain from equation (32), which sets the current limit of element F6.

F_formula = -LIMIT(@lin,@LO,@HI),

F_formula = LIMIT(@lin,@LO,@HI),

$H I=F 5 @ f u n c r e f(@ g a i n)$,

$L O=-@ H I$,

HI=F6@funcref(@gain),

.FUNC F5@funcref(g)

$=\{$ TABLE $(\mathrm{g}, 1,300 \mu, 2,250 \mu, 3,200 \mu, 4,150 \mu$,

$5,100 \mu, 6,75 \mu, 7,50 \mu, 8,30 \mu)\}$,

.FUNC F6@funcref(g)

$=\{$ TABLE $(\mathrm{g}, 1,300 \mu, 2,250 \mu, 3,200 \mu, 4,150 \mu$,

$5,100 \mu, 6,75 \mu, 7,50 \mu, 8,30 \mu)\}$.

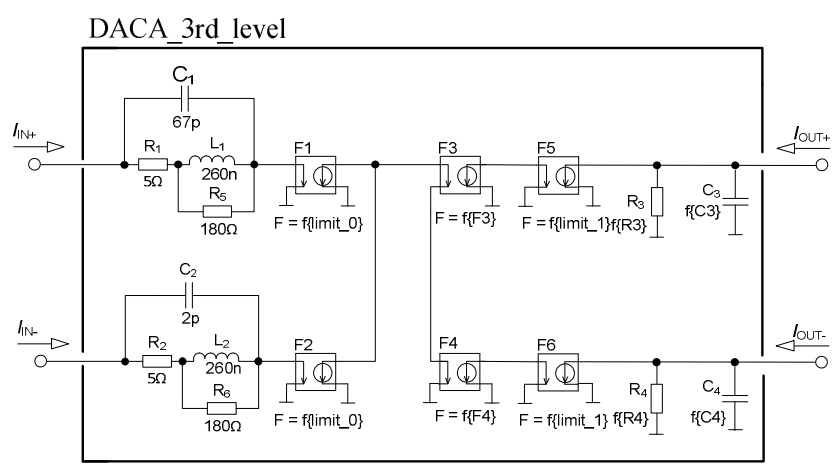

Fig. 8. Model of the third level of DACA element

The functions of the input and output current limit are demonstrated in the graph in Fig. 9. The input current, connected to IN-, is the harmonic signal, which is indicated in the graph as $\mathrm{I}_{\mathrm{IN}}$ and has the following parameters: amplitude $1 \mathrm{~mA}$ (intentionally too high for DACA element in order to show the limitations), frequency of $1 \mathrm{kHz}$. Gain of DACA is set to gain $=2$. Input current after the limit element is represented by the curve marked as LIMIT $\mathrm{I}_{\mathrm{IN}}$. The amplitude of this curve is limited to $300 \mu \mathrm{A}$, according to the set values of upper and lower input current limit. Curves $\mathrm{I}_{\text {OUT+ }}$ and $\mathrm{I}_{\text {OUT- }}$ represent output currents of the active element, their amplitude is limited to $250 \mu \mathrm{A}$ and corresponds to the values of equations (31) and (32) for the current limit gain value equal to two. Furthermore, the graph in Fig. 9 shows the particular gain limit represented as GAIN LIMIT $\mathrm{I}_{\mathrm{IN}}$ (gain is 2 in this particular case). This curve is based on the gain parameter defined in equation (18) for a gain value (variable gain) equal to two and the input current limit of element F1. 


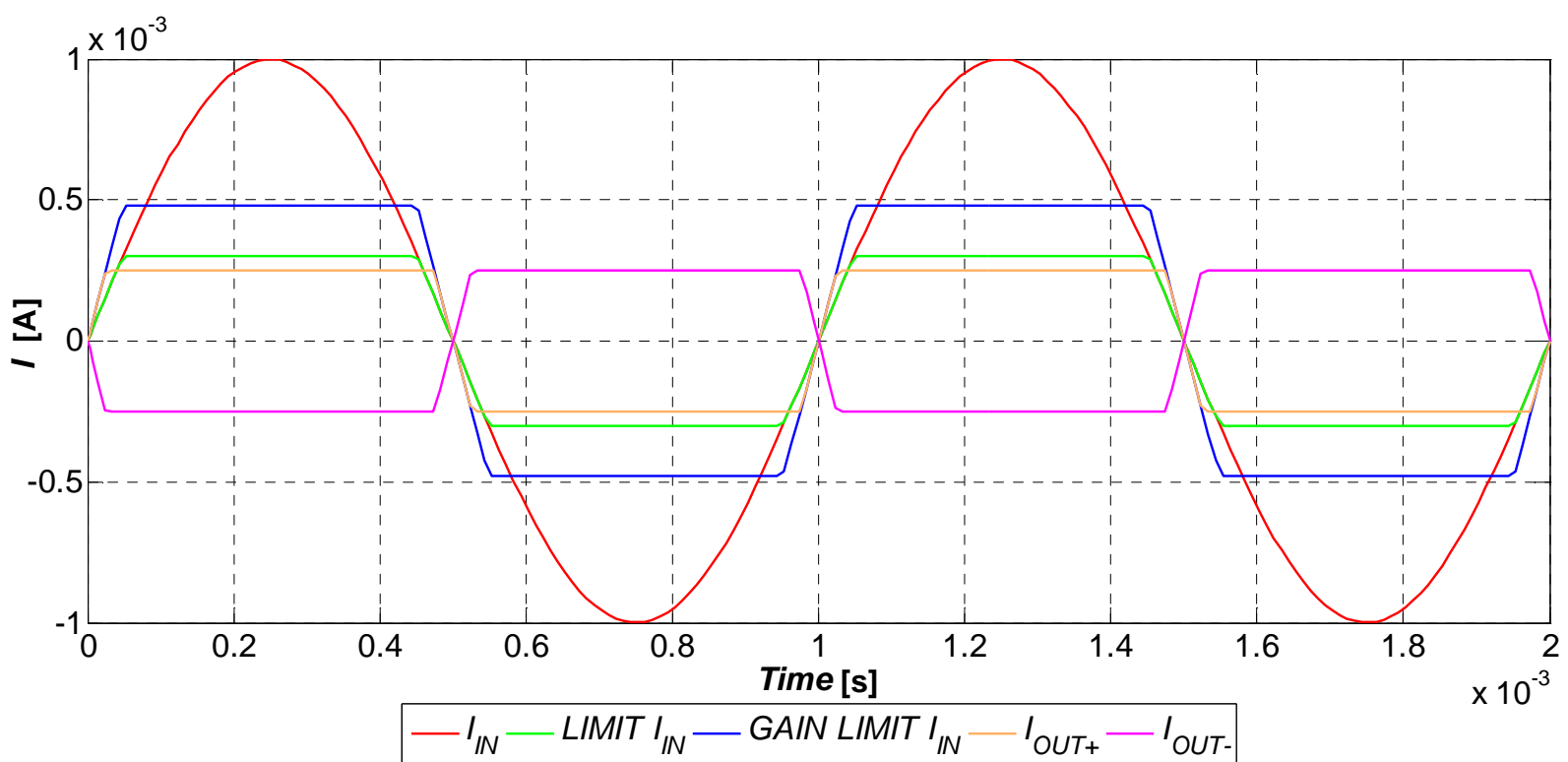

Fig. 9. Demonstration of current limitations in the third level of model in time domain

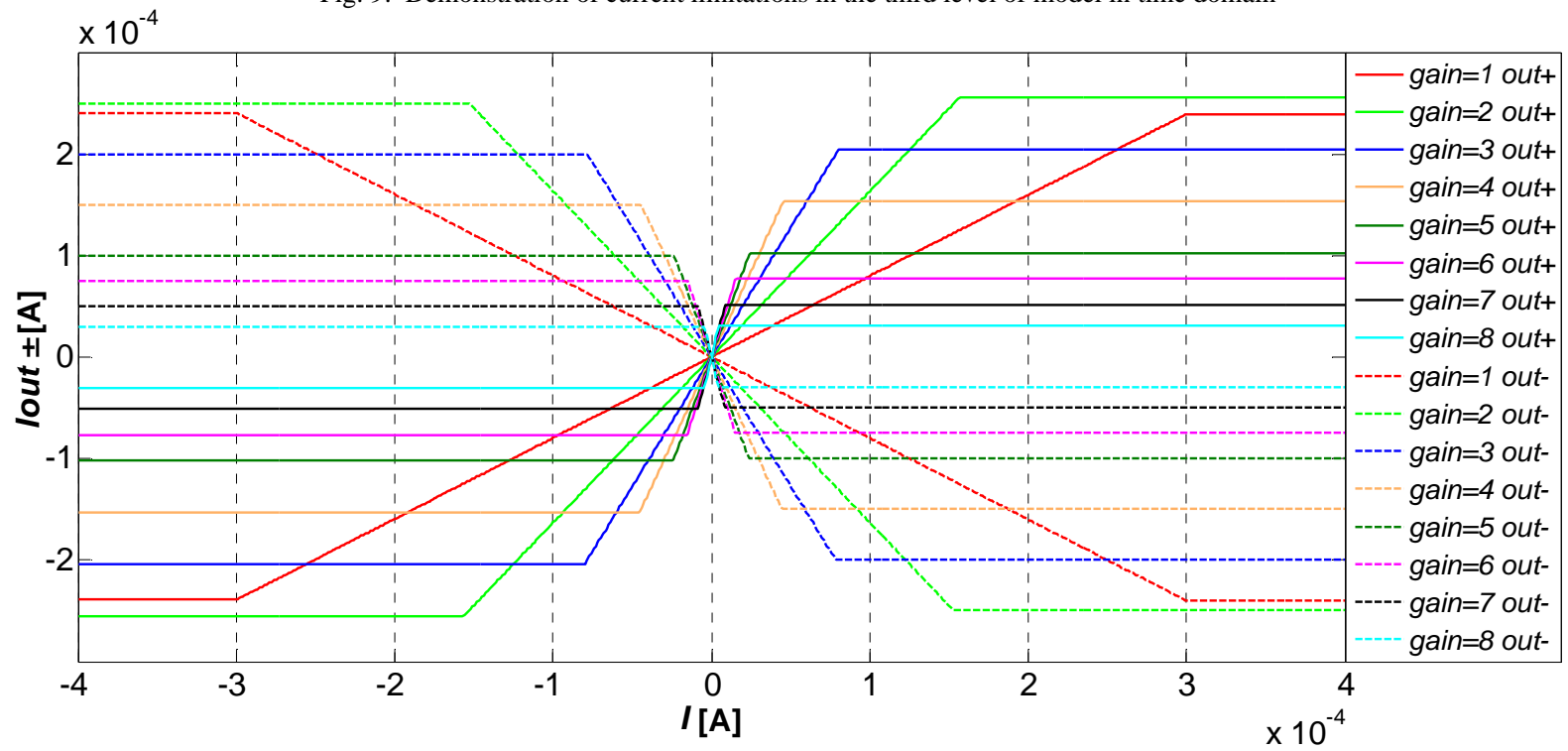

Fig. 10. Demonstration of current limitation for each value of gain in DC/DC graph

Fig. 10 shows the DC characteristics of the input current

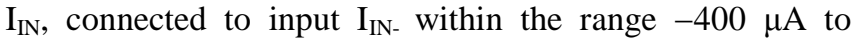
$+400 \mu \mathrm{A}$. The output currents for both outputs OUT + and OUT - show the characteristic tendency for specific gain and for each gain value the current limit of elements F5 and F6 is reflected, according to equations (26) and (27). Minor deviations of the inverting and non-inverting output correspond to the behaviour of these outputs in a real active element DACA. The graph further clearly indicates that the current limit decreases with increasing gain.

\section{COMPARISON WITH RESULTS FROM PRACTICAL MEASUREMENT}

Numerical values presented in the ABM model were obtained through thorough measurements and subsequent analysis of the results of $\mathrm{DC}, \mathrm{AC}$, and impedance characteristics for eight of produced DACA chips [17]. Figure 11 shows the curves for all eight values of gain depending on frequency for both measurement and simulation model results (real DACA and ABM simulation model of DACA). When comparing curves in case of the graph in Fig. 11, there are some deviations of the ABM model from real element at higher gain values. The graph in Fig. 12 shows the frequency dependence of the output impedance for all eight values of gain again for ABM simulation model and real DACA element. When comparing curves in Fig. 12, we can observe nearly identical curves for the output impedance for the model DACA and the real element DACA. In the graph in Fig. 13 the input impedance corresponds to value $Z_{\mathrm{IN}}=5 \Omega$ up to $1 \mathrm{MHz}$, for the model DACA and the real element DACA. 


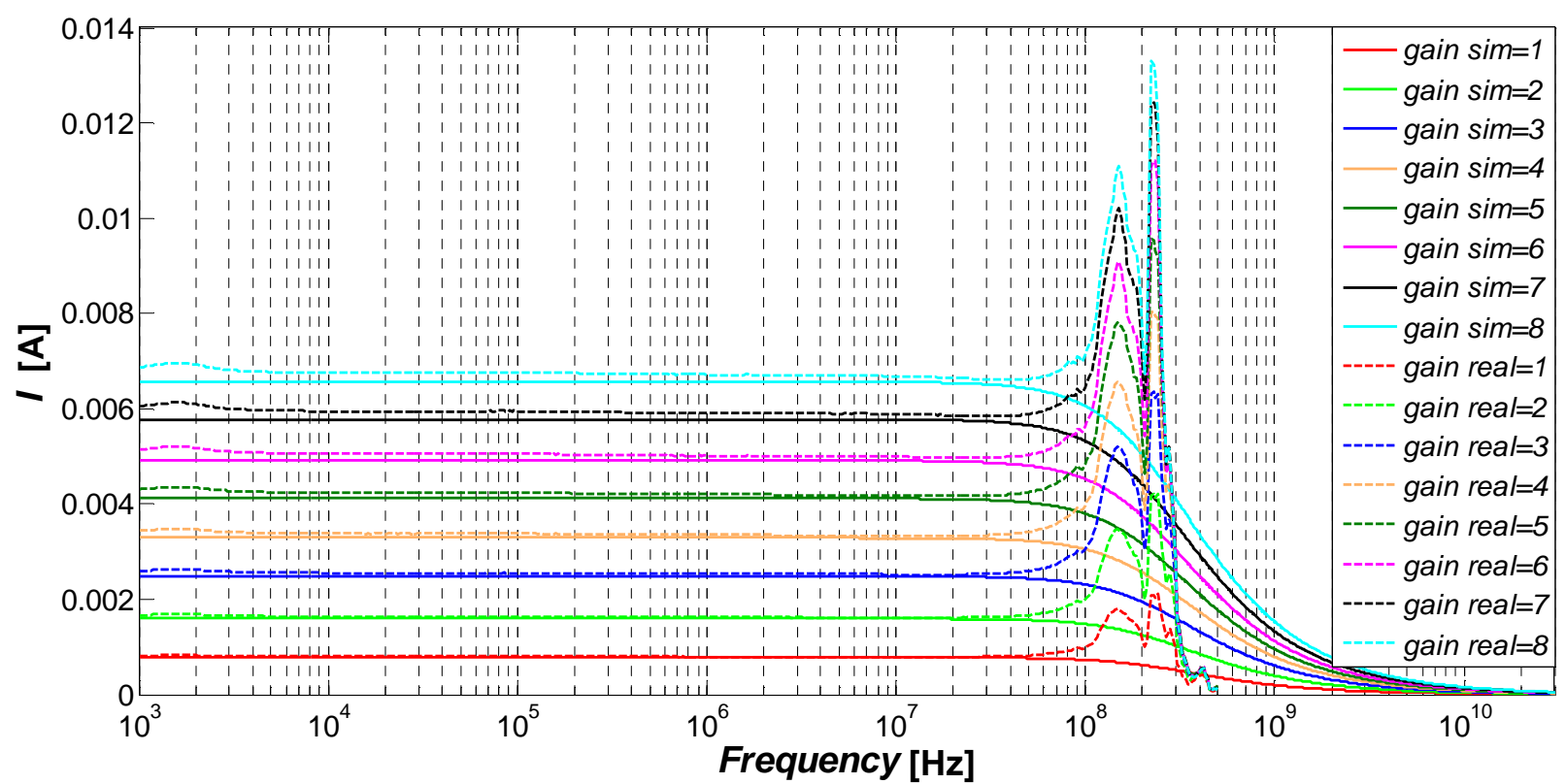

Fig. 11. Comparison of measured and simulated gain for all variants of gain in DACA

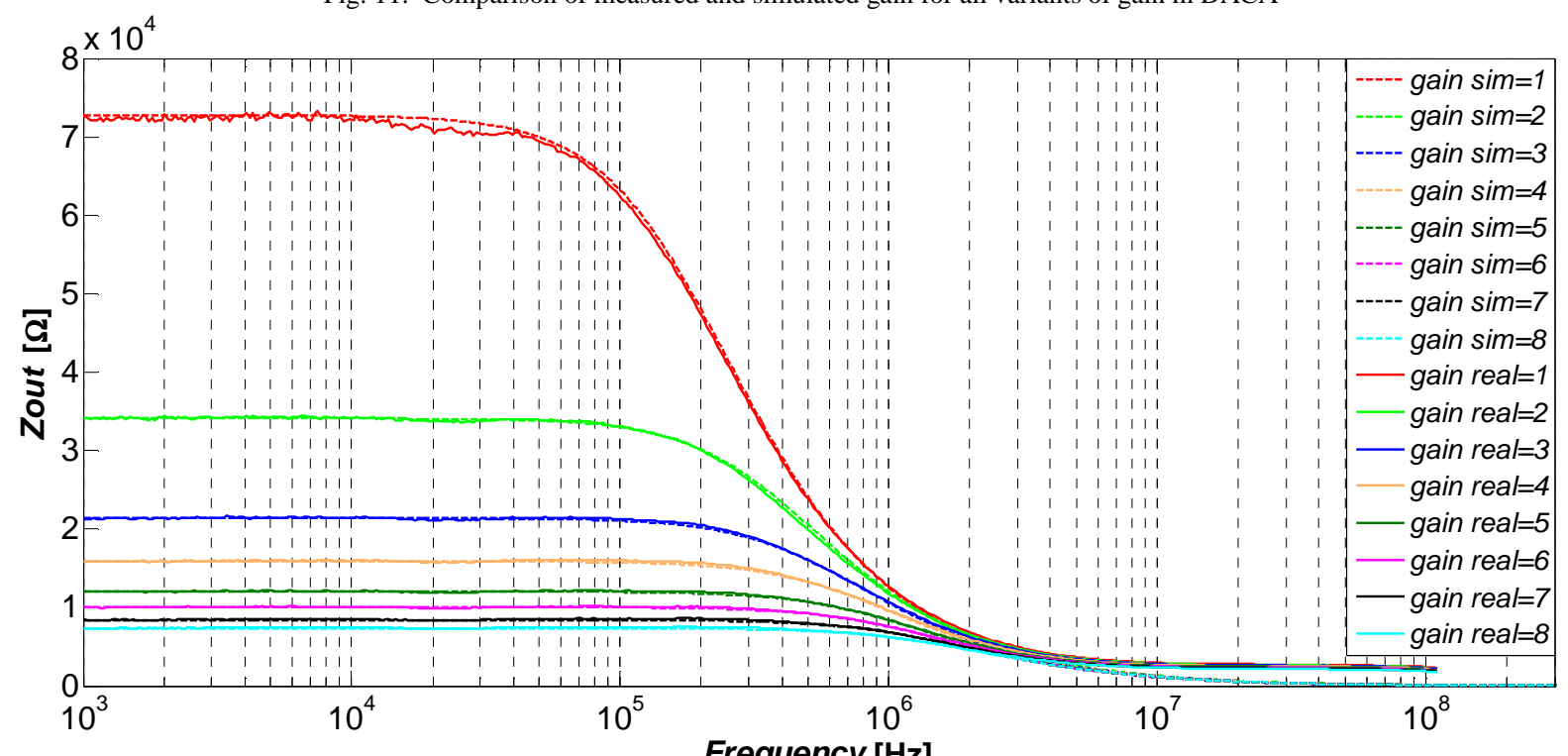

Frequency [Hz]

Fig. 12. Comparison of measured and simulated output impedance for all variants of gain in DACA

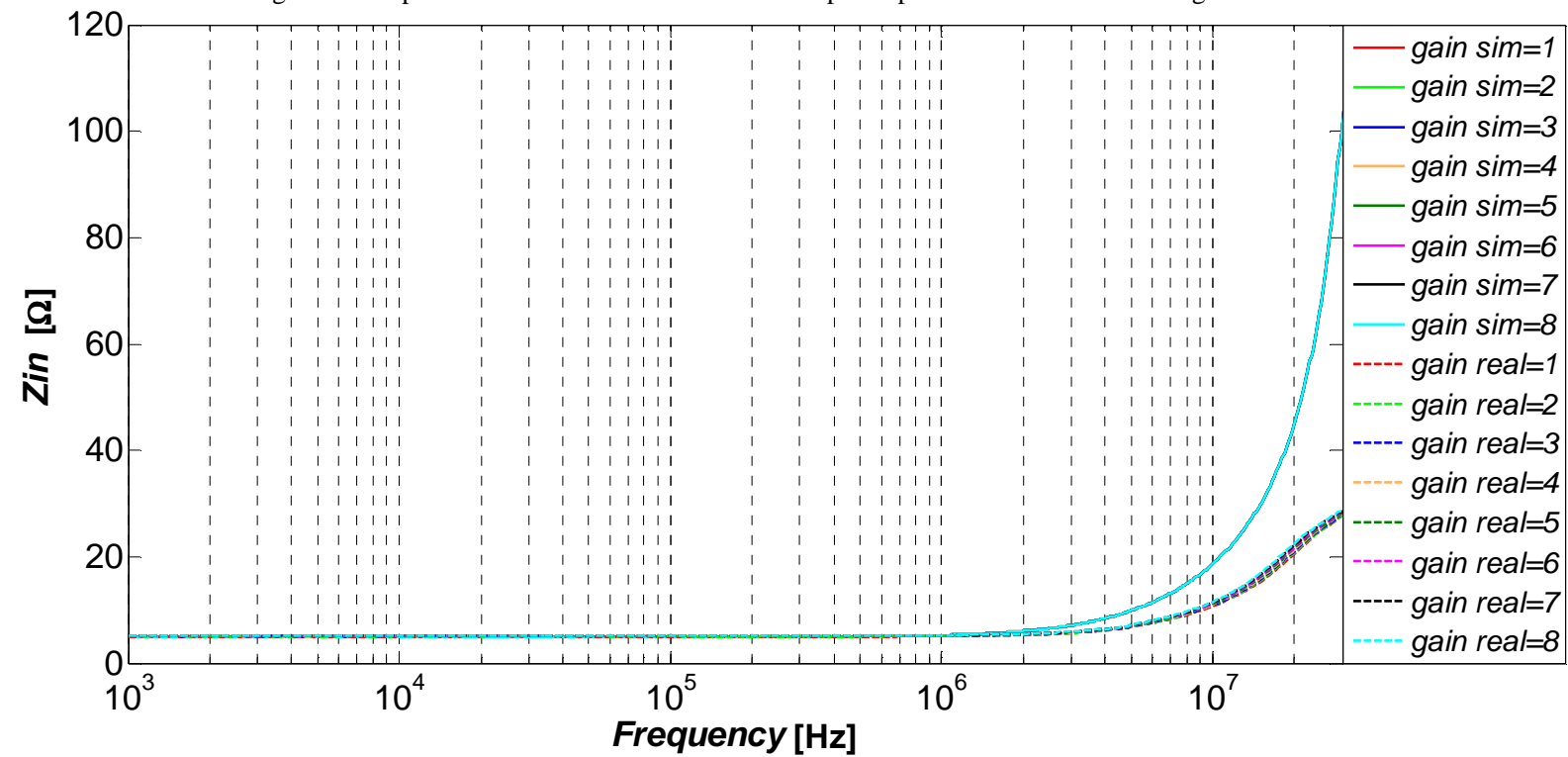

Fig. 13. Comparison of measured and simulated input impedance for all variants of gain in DACA 


\section{CONCLUSION}

In this paper we presented an ABM model of recently developed digitally controlled current amplifier DACA. The ABM model of the active element is defined in four levels, each of which adds certain behavioural characteristics of the active element. The zero level is designed as a completely ideal and involves only an adjustment of gain from one to eight with a unity step. Gain selection is done by setting the value of the variable amplification (gain) from the specified range. The first level is based on the zero level, but the ideal gain values are replaced by real values and the level function reflects the influence of the input impedance (set to be constant; $5 \Omega$ ) and the output impedance (impedance value depends on the selected gain). The second level reflects the frequency dependence of the gain as well as frequency dependence of the input and output impedance. The third level of this model additionally reflects the input current limits (constant current limit value of $300 \mu \mathrm{A}$ ) and output current limit (particular limit value depends on the specified gain). These models are suitable to performance the initial simulations, where the operability of the proposed function blocks using DACAs as active elements is evaluated. Based on the simulation results, proper optimation steps can be done before experimental measurements are performed. Selected characteristics of the model and the real element DACA are presented in the form of graphs and are compared to each other.

\section{ACKNOWLEDGMENT}

Research described in the paper was financed by the National Sustainability Program under grant LO1401 and by Czech Science Foundation project under grant No. GP14$24186 \mathrm{P}$ and by internal grant No. FEKT-S-14-2352. For the research, infrastructure of the SIX Center was used.

Authors would like to thank to prof. Zdenek Kolka for his help in the stage of ABM preparation.

\section{REFERENCES}

[1] D. Biolek, R. Senani, V. Biolkova, Z. Kolka, "Active elements for analog signal processing: classification, review, and new proposals," Radioengineering, vol. 17, 2008, pp. 15-32.

[2] C. Acar, S. Ozoguz, "A new versatile building block: current Differencing buffered amplifier," Microelectronics Journal, vol. 30, 1999, p. 157-160.

[3] S-I. Liu, "High input impedance filters with low component spread Using current-feedback amplifiers," Electron Lett, vol. 31, 1995, p. $1042-1043$.

[4] Analog Devices, "Linear Products Data Book," Norwood, MA, 1990.

[5] Analog Devices, "AD844: $60 \mathrm{MHz}, 2000 \mathrm{~V} / \mu \mathrm{s}$ Monolithic Op Amp Data Sheet", Rev F, 2009.

[6] D. Biolek, "CDTA - Building Block for Current-Mode Analog Signal Processing," In: Proceedings of the ECCTD03. Krakow (Poland), vol. III, 2003, pp. 397-400.

[7] N. A. Shah, M. Quadri, S. Z. Iqbal, "CDTA based universal transadmittance filter," Analog Integr. Circ. Sig. Process. Journal, 2007, pp. 65-69.

[8] N. Herencsar, J. Koton, K. Vrba, J. Misurec, "A Novel Current- Mode SIMO Type Universal Filter Using CFTAs," Contemporary Engineering Sciences, 2009, vol. 2, s. 59-66, ISSN: 1313-6569.

[9] M. Siripruchyanun, W. Jaikla, "Current controlled current conveyor transconductance amplifier (CCCCTA): a building block for analog signal processing," In International Symposium on Communications and Information Technologies, Sydney (Australia), 2007, p. 209-212.
[10] R. Prokop, V. Musil, "New modern circuit block CCTA and some its applications," in Proc. of the $14^{\text {th }}$ International Scientific and Applied Science Conference Electronics - ET 2005, 2005, pp. 93-98.

[11] A. Fabre, O. Saaid, F. Wiest, C Boucheron, "High frequency applications based on a new current controlled conveyor," IEEE Trans Circuits Syst I: Fundam Theory Appl, vol. 43, 1996, p.82-91.

[12] N. Herencsar, J. Koton, K. Vrba, A. Lahiri, O. Cicekoglu, "Currentcontrolled CFTA-based current-mode SITO universal filter and quadrature oscillator," In: Proceedings of 2010 International Conference on Applied Electronics - APPEL,2010, p. 121-124.

[13] A. A. El-Adawy, A. M. Soliman, H. O. Elwan, "Low voltage digitally controlled CMOS current conveyor," International Journal of Electronics and Communication, vol. 56, 2002, no. 3, p. 137-144, DOI: $10.1078 / 1434-8411-54100086$.

[14] W. Tangsrirat, K. Klahan, T. Dumawipata, W. Surakampontorn, "Low-voltage NMOS-based current differencing buffered amplifier and its application to current-mode ladder filter design," Int. J. Electron., vol.93, 2006, p.777-791.

[15] D. Prasertsom and W. Tangsrirat, "CMOS Digitally Controlled Current Follower and Its Application," In: Proc. of the 12th International Symposium on Singapore, 2005, pp. 486-489.

[16] J. Koton, N. Herencsar, K. Vrba, J. Jerabek, "Digitally Adjustable Current Amplifier and its Application in Fully Differential CurrentMode Band- Pass Filter Design," Internet Journal Elektrorevue, vol. 90, 2010, p. 1-6, ISSN: 1213-1539.

[17] J. Jerabek, K. Vrba, I. Koudar, "Dual Wide-Bandwidth Digitally Adjustable Current Amplifier (DACA)," Data Sheet, 2010, online: http://www.utko.feec.vutbr.cz/ jerabekj/daca.pdf

[18] J. Jerabek, R. Sotner, K. Vrba, "Fully-differential current amplifier and its application to universal and adjustable filter," International conference on applied electronics(AE 2010), 2010, p. 141-144.

[19] J. Jerabek, K. Vrba, "Current- mode tunable and adjustace filter with digitally adjustace current amplifier and transconductance amplifiers," In: Proceedings of the European Conference of Circuits Technology and Devices (ECCTD'2010), NAUN, 2010, p. 101-104, ISBN: 978960-474-250- 9

[20] L. Langhammer, J. Jerabek, "Fully Differential Universal CurrentMode Frequency Filters Based on Signal- Flow Graphs Method," International Journal of Advances in Telecommunications, Electrotechnics, Signals and Systems, vol. 1, 2014, p. 1-12, ISSN: 1805- 5443.

[21] J. Jerabek, J. Koton, R. Sotner, K. Vrba, "Comparison of Fully Differential and Single-Ended Current-Mode Band- Pass Filters with Current Active Elements", In Proceedings of 7th International Conference on Electrical and Electronics Engineering - ELECO 2011, Bursa, Vol. 2, 2011, p. 100-104.

[22] E. Tlelo-Cuautle, E. Martínez-Romero, C. Sánchez-López, F. Fernández, S. Tan, P. Li, M. Fakhfakh," Behavioral Modeling of Mixed-Mode Integrated Circuits, Advances in Analog Circuits,“ In Tech, 2011, ISBN: 978-953-307-323-1.

[23] H. Du, D. Gorcea, P. P. M. So, W. J. R. Hoefer, “A SPICE analog behavioral model of two-port devices with arbitrary port impedances based on the S-parameters extracted from time-domain field responses," International Journal of Numerical Modeling: Electronic Networks, Devices and Fields, vol. 21, 2007, p. 77-79. 